\title{
Leakage versus Material Filtration in Barrier Facemask Efficiency
}

\author{
Ali Bahloul ${ }^{1,2 *}$, Clothilde Brochot ${ }^{2}$, Barbara Layne ${ }^{3}$ \\ ${ }^{1}$ Chemical and Biological Hazard Prevention, Institut de recherche Robert-Sauvé en santé et en sécurité du travail, Montréal, \\ Canada \\ ${ }^{2}$ Department of Building, Civil and Environmental Engineering, Concordia University, Montréal, Canada \\ ${ }^{3}$ Department of Studio Arts, The Milieux Institute for Art, Culture and Technology, Concordia University, Montréal, Canada \\ Email: ^ali.bahloul@irsst.qc.ca
}

How to cite this paper: Bahloul, A., Brochot, C. and Layne, B. (2021) Leakage versus Material Filtration in Barrier Facemask Efficiency. Health, 13, 439-453. https://doi.org/10.4236/health.2021.134035

Received: March 6, 2021

Accepted: April 24, 2021

Published: April 27, 2021

Copyright (c) 2021 by author(s) and Scientific Research Publishing Inc. This work is licensed under the Creative Commons Attribution International License (CC BY 4.0).

http://creativecommons.org/licenses/by/4.0/ (c) (i) Open Access

\begin{abstract}
The World Health Association and many governmental agencies recommend the wearing of facemasks by the general public to prevent the spread of COVID-19. It is believed that masks can significantly protect others and may offer some protection to the wearer. Although there are standards for FFRs, surgical masks, and recently for barrier face coverings, they all indicate the level of protection for the wearer. However, testing facial masks not at the point of inhalation, but at the source, the exhale, offers a new perspective on how to impede particle emissions. In this paper, the experimental results show that, although the barrier face covering is less effective than FFRs or surgical masks, it can reduce the concentration of aerosols downstream of the device. The results on barrier efficiency show a rapid decrease in effectiveness when the face covering is not sealed to the head. The barrier efficiency of two of the barrier face coverings tested is strongly dependent on leakage caused by the fit rather than the material. While some materials certainly are more effective than others in inhibiting particle penetration, an even more profound factor is the amount of leakage emitted from a mask. New approaches to fit and design in order to create a seal against leakage will become an important factor in combatting SARS-CoV-2.
\end{abstract}

\section{Keywords}

COVID-19, Facemask, Filtration, Leakage, Aerosol, Airborne Transmission

\section{Introduction}

Following numerous calls from the scientific community to outline evidence on the route of airborne transmission of SARS-CoV-2 [1] [2], the World Health 
Organization (WHO) [3] has updated its information on the routes of transmission of SARS-CoV-2 to integrate this new information into the two other transmission routes: contact and droplet transmission route. Facial masks are a key component of most reopening plans with some governments making masks obligatory in public spaces [4] [5] [6]. Recent findings [7] demonstrate that the mandating of face coverings for use in public spaces has been associated with a decline in the daily COVID-19 growth rate in selected locations in the USA. This suggests that hundreds of thousands of cases may have been averted by these directives.

In the light of this recommendation, one must first define the different "masks" that can be used during this pandemic period. It can be classified according to their use: some are designed to protect the wearer from environmental exposure (filtering facepieces respirators, FFRs), while other face coverings protect individuals from the wearer's potential emissions. FFRs are disposable respiratory protection devices intended to protect the user against inhalation of harmful agents (such as aerosols). This type of mask is generally used in workplaces. In Canada [8], the wearing of medical masks is mainly recommended in healthcare establishments. On the other hand, face covering masks are designed to limit the spread of germs by the wearer in their environment, primarily by retaining respiratory droplets.

FFRs and surgical masks are devices widely used before the pandemics are subjected to standards testing before they are released to the market. For example, in North America, N95 FFRs are regulated by 42 CFR Part 84 [9]. This standard establishes performance via the filtration efficiency and the pressure drop measurements. In this study, the authors refer to the NIOSH certification as a reference. Also, fit tests are required to choose the best FFR considering the wearer's face. However, no fit testing is mandated for medical masks.

Some papers are based on the classical efficiency measurement method (i.e. applied according to standards) in an attempt to estimate the effectiveness of these facemasks [10] [11] [12] [13]. They show that wearing a mask reduces the amount of aerosol generated downstream of the mask. However, the effectiveness is generally lower than the N95 FFRs or surgical mask efficiencies. Some researchers have proposed new methodologies to measure the material's efficiency or the mask's performance [14] [15] [16]. However, these methodologies either count particles larger than 0.5 micrometer or have been tested in a non-controlled environment.

Homemade facemasks or barrier face coverings are simple cloth face coverings that can be made at home and can help prevent the spread of COVID-19. A plethora of commercial and homemade facemasks appeared widely during the pandemic, and many tutorials for making facemasks could be found in different countries [17] [18]. It is still unknown which facemasks are most beneficial as there are countless variables in materials and designs.

The ASTM F3502-21 [19] standard, recently published in 2021, describes the 
specifications for barrier face coverings. This standard presents the methodology for testing filtering efficiency of the material. This method is generally applied to the assessment of inhalation efficiency and not exhalation efficiency. Indeed, the standard does not take into account the protection efficiency via the emitter wearing the barrier mask. Also, the fit factor is not considered in this standard.

The question here is whether a classical methodology is applicable to facemasks. In addition to efficiency and pressure drop, the fit of the mask to the head could be a predominant parameter for facemasks. Leaks from around the outer edges of the mask drastically decrease mask efficiency. Measurements on respiratory protection devices have shown that this decrease becomes greater in relation to increased amounts of leakage [20] [21] [22]. Van der Sande et al. [23] and Davies et al. [24] determined that facemasks provided about half the protection of standard surgical masks. Their results also demonstrate the importance of the fit. In the case of respiratory protective devices, respiratory protective device functions at negative pressure. In the case of facemasks, emission takes place under positive pressure that may increase the role of leaks in the wearer's emission. Since it is generally considered that wearing a facemask is essentially to protect others, the scientific community should find a standard methodology to assess and qualify them. What is the relationship between the efficiency of materials used in masks and controlling particle leakage that may be expressed through the sides or other points of escape inherent to a mask's design? Testing that measures the amount of particle escape in various mask scenarios leads to a clearer understanding of how the virus may enter the environment, providing new insights on how microorganism containment might best be achieved.

The goal is to determine the effects of the material filtration properties versus the effect of leakage related to facemask fit or holes in the design on the barrier efficiency. To determine how the environment may influence the results, tests are conducted in both a ventilated room and a controlled chamber. Different conditions are analyzed according to the environment, the filtration and air resistance properties of the facemask, the inhalation flowrate of the person to protect and the leakage due to the fitting of the mask. Two manikin heads are used, one head simulates the emitter (exhalation), the other acts as the receptor (inhalation). Barrier efficiency consists of an evaluation of the performance of the facemask in stopping projectiles from the wearer's mouth simulating the droplets generated by coughing, sneezing, exhalation, and speaking. Particle sizes smaller than $1 \mu \mathrm{m}$ are used, representing the worst-case scenario since they are likely to pass through the facemask material and also escape as leakage between the facemask and the manikin head.

\section{Materials and Methods}

\subsection{Facemasks}

Three different facemasks are used during this study (Figure 1): two commercially available facemasks and one homemade mask made of one-layer of woven 
cotton and designed according to an online pattern. The facemasks are labelled as the "white facemask", "blue facemask" and the "green facemask". Each is tested inside a chamber under controlled conditions (Figure 2(b)).

\subsection{Facemasks' Characteristics}

The characteristics of the three facemasks used in this study are presented and compared in Table 1. The white facemask contains an electret medium, whose fibers are electrostatically charged. These charges allow the particles to be collected with an additional collection mechanism of electrostatic forces. The blue facemask contains a mechanical medium, collecting the particles through sedimentation, interception, inertial impaction and Brownian diffusion.

\subsection{Facemasks' Filtration Efficiency and Pressure Drop}

In this section, the filtration characteristics of the materials of the three facemasks are determined by using the classical methodology on an existing setup for measuring the performance of respiratory protection devices [24] [25] [26] [27] [28]. Figure 3(a) and Figure 3(b) respectively show the initial filtration efficiency and the pressure drop of the three facemasks at the receptor inhalation flowrate of $85 \mathrm{~L} / \mathrm{min}$. One can note that a certified N95 FFR must have a minimum
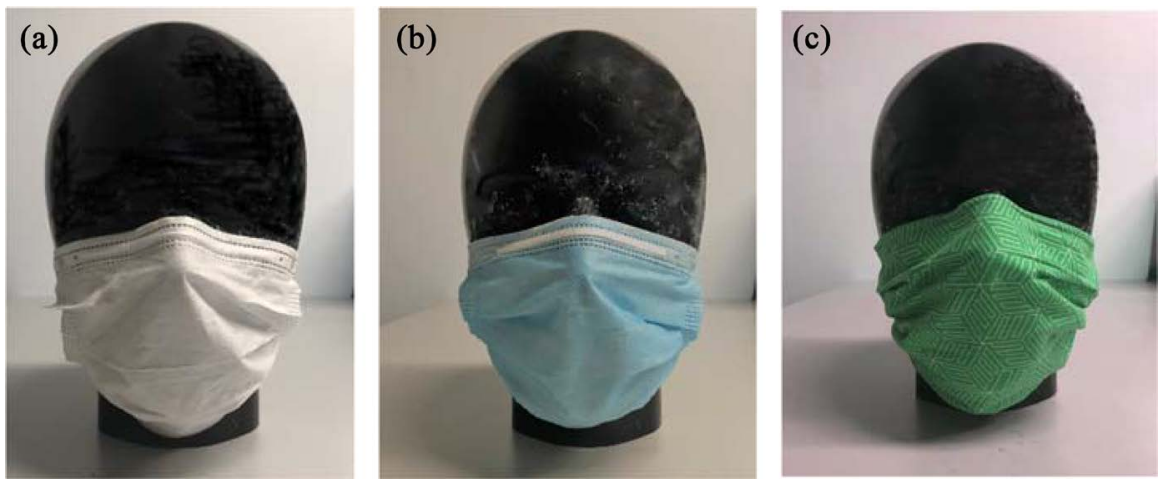

Figure 1. Three facemasks used in study: (a) white facemask, (b) blue facemask, and (c) green facemask.

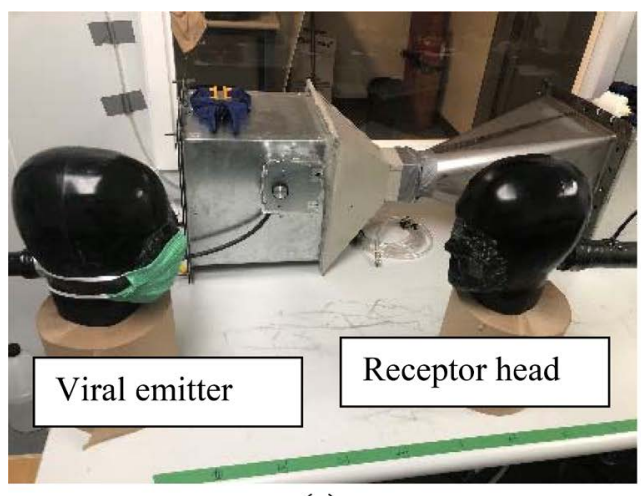

(a)

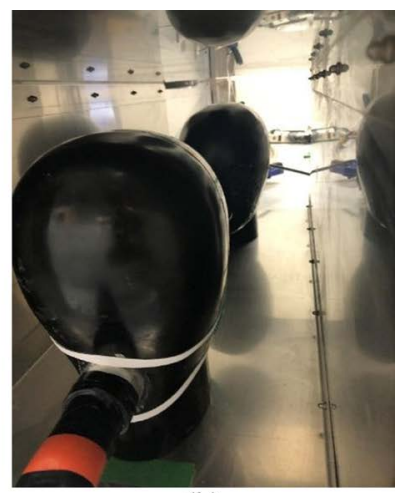

(b)

Figure 2. Measuring barrier efficiency (a) inside a ventilated room and (b) inside the controlled environmental chamber. 
Table 1. Characteristics of facemasks.

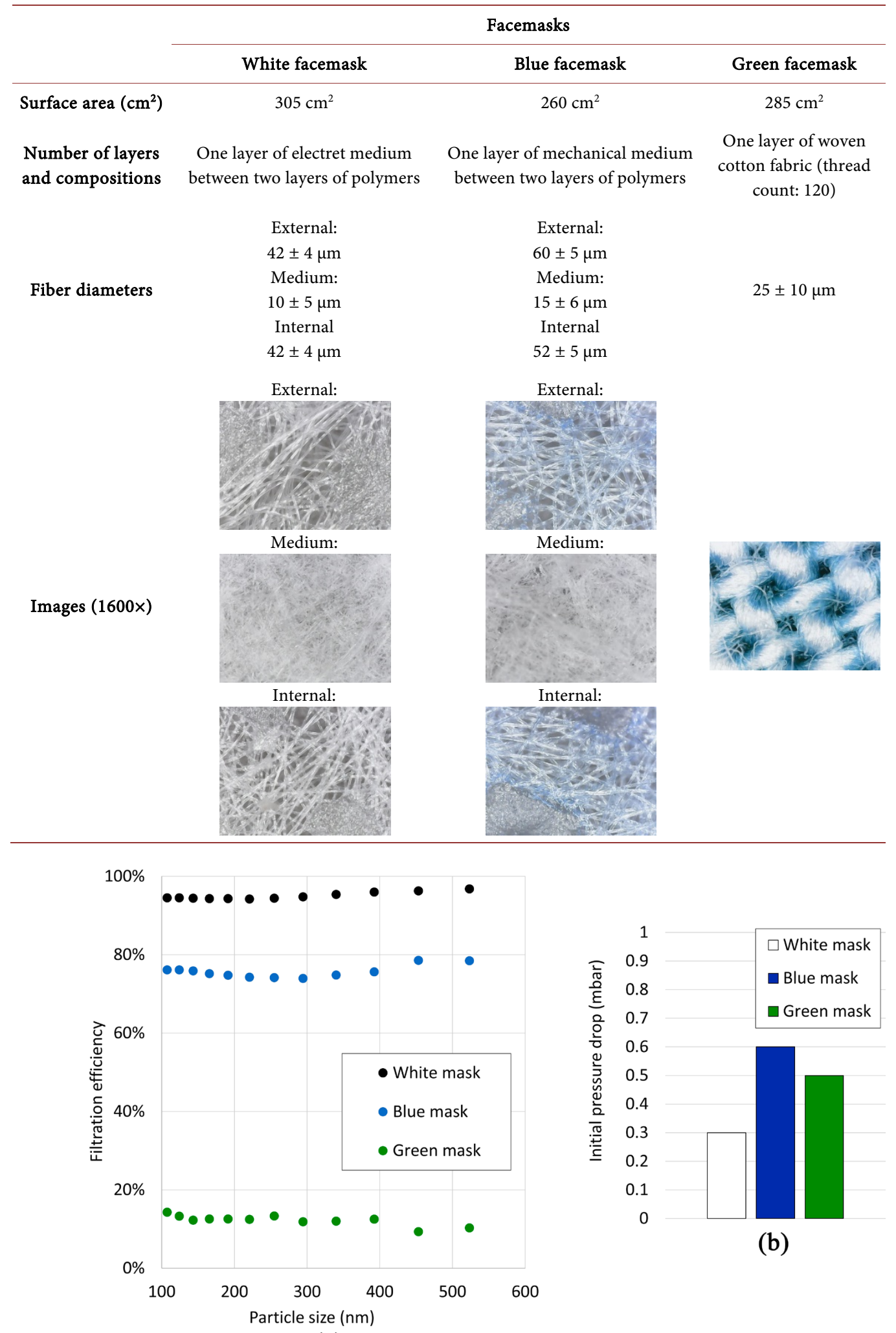

(a)

Figure 3. Material properties of the three facemasks: (a) filtration efficiency according to particle size and (b) pressure drop. 
filtration efficiency of $95 \%$ and a maximum pressure drop of 3.43 mbar. One then plays between the two parameters to have a good efficiency while having an acceptable breathability for the wearer.

The white facemask material has a filtration efficiency of more than $90 \%$ and a low pressure drop of 0.3 mbar. The white facemask made of fiber is then very advantageous because it has good filtration efficiency and good breathing comfort.

The blue facemask material has a filtration efficiency of around $80 \%$ and a pressure drop of 0.6 mbar. The filtration efficiency and the breathing comfort associated with the blue facemask material are also acceptable.

Finally, the green facemask has a low filtration efficiency of less than $20 \%$ and pressure drop of 0.5 mbar. However, this resistance increases rapidly during the test. Clogging of the fabric material occurs on the surface and thus makes it uncomfortable with use.

\subsection{Aerosol Generation}

A wet air jet containing $\mathrm{NaCl}$ particles is installed at the mouth of the exhalation head using an orifice with an air expiration speed of $6 \mathrm{~m} / \mathrm{s}$. According to previous studies, this can be considered as an extreme case. Respiration and speech generate jet speeds rarely exceeding $5 \mathrm{~m} / \mathrm{s}$ [29] and mainly expel small droplets. Violent expiratory events such as coughing and sneezing generate turbulent jets with higher maximum speeds [29] [30] [31]. Experimental studies are still needed, in particular on the size distribution and velocities produced by sneezing. Humid $\mathrm{NaCl}$ particles are used to simulate droplets coming from head-exhalation. These particles are generated by a 6-jet collision (BGI) based on an $\mathrm{NaCl}$ solution using a flowrate of $15 \mathrm{~L} / \mathrm{min}$. The aerosol generation follows the NIOSH standard. The lognormal distribution has a mean count diameter around $70 \mathrm{~nm}$ and a geometric standard deviation lower than 1.86. Although particles of size around 100 $\mathrm{nm}$ cannot reflect the whole generated bioaerosol, it represents the "worst case scenario".

\subsection{Measurement Method}

A Scanning Mobility Particle Sizer (SMPS) is used to measure the concentration of $\mathrm{NaCl}$ particles inhaled by the receptor. The aerosol is collected by a probe inside the head. It is then selected according to its electric mobility diameter, by the electrostatic classifier used with a Long Differential Mobility Analyzer (Long-DMA TSI 3082) and the concentration is measured by a Condensation Particle Counter (CPC TSI 3752). Barrier efficiency is defined by the effectiveness of the facemask worn by the emitter head in stopping the transmission of the simulated virus to the receptor head. The measurement was carried out at the beginning of the experiment, before any possible clogging effect and facemasks were not conditioned before their use. As the authors use the 42 CFR Part 84 standard as a reference, the barrier efficiency measurements are performed at 
a constant flow rate of $85 \mathrm{~L} / \mathrm{min}$.

Barrier efficiency $\left(E_{\mathrm{barrier}}\right)$ is then obtained via the ratio of average concentrations, expressed in number, with and without using facemasks using the following Equation (1). The results presented in this paper are the mean of three measurements.

$$
E_{\text {barrier }}=1-\frac{C_{\text {with mask }}}{C_{\text {without mask }}}
$$

First, the barrier efficiency of one facemask is analyzed in a ventilated room with negative pressure (Figure 2(a)). and then in a controlled environment chamber (Figure 2(b)). Based on this comparison it was decided to perform the experiments in the controlled environment chamber to limit the variables to only the properties of the facemask with no environmental influences.

\subsection{Barrier Efficiency in a Ventilated Room}

The ventilation room is a negative pressure chamber (controlled at -20 mbar) 5 meters long, 3 meters wide and approximately 2.5 meters high. An air supply plenum is installed in the center of the chamber ceiling and an outlet plenum is located at 0.2 meters from the floor. The two heads are installed on a table facing one another (Figure 2(a)).

These first tests were carried out on the homemade green facemask in a ventilated room with negative pressure. Figure 4 presents the barrier efficiency according to the particle diameter. "One green face" facemask curve is calculated when the emitter head wear a facemask and the "two green facemasks" curve is calculated when the two heads wear a facemask. Wearing a facemask by both manikin heads thus increases the barrier efficiency. It can be noted that the ventilation

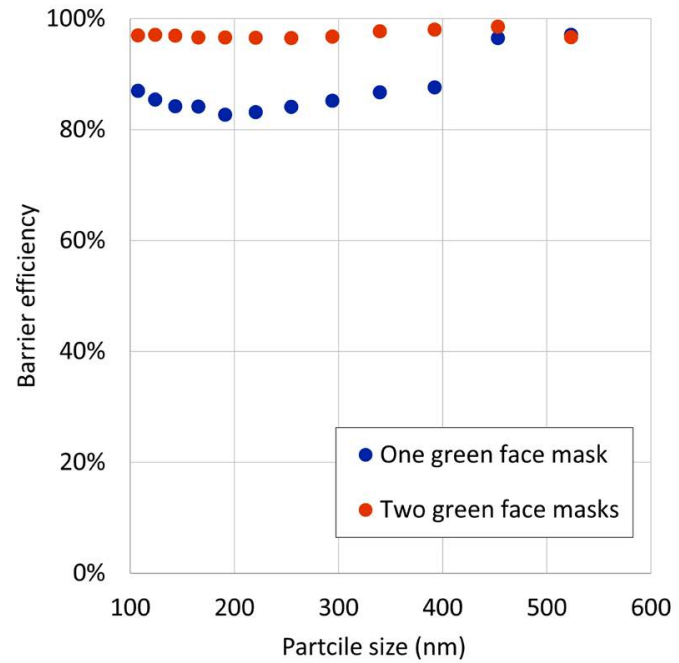

(a) (b) One green facemask

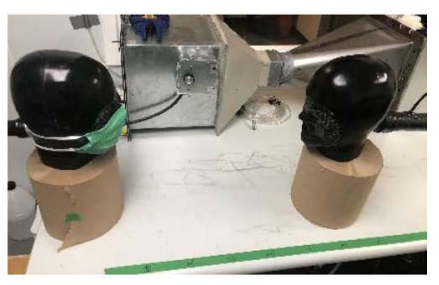

(c) Two green facemasks

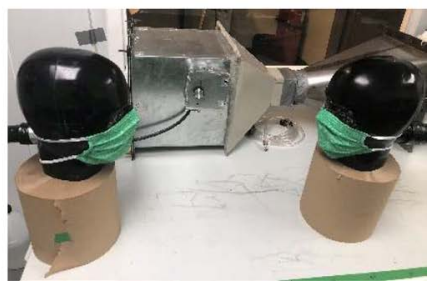

Figure 4. (a) Barrier efficiency of the homemade green facemask inside the ventilated room, when the emitter head wears a facemask (b) and when both the emitter and the receptor heads wear a facemask (c). 
and the presence of structures in the room could influence the barrier efficiency of the facemask.

\subsection{Barrier Efficiency in the Controlled Environment Chamber}

The controlled environment chamber consists of a vein with a section of $30.5 \mathrm{~cm}$ $\times 30.5 \mathrm{~cm}$ ( 1 foot $\times 1$ foot), and approximately $50 \mathrm{~cm}$ in length, installed in the ventilated room. Considering the walls of the vein and its length, one can consider that this experiment represents an unrealistic situation, or at least a "worst case scenario". This configuration was actually chosen to facilitate the measurement and characterize the barrier efficiency in the different configurations tested. Two configurations are then used:

1) The two heads are installed in the controlled environment chamber. A facemask is installed on the emitter head. This configuration permits to study the leakages related to the fit of the facemask (Figure 2(b)).

2) Instead of the emitter head, a $2.5 \mathrm{~cm}$ diameter tube is used to hold a circular piece of material (Figure 5). Assuring a complete seal around the perimeter of the fabric, this configuration provides a better control of the amount of leakage by creating $1.5 \mathrm{~mm}$ holes with an electric drill.

In the following test, two manikin heads were placed in the controlled environment chamber to isolate the heads (Figure 2(b)). Only the emitter head wears a facemask (the green homemade facemask).

\subsection{Calibration Tests in the Controlled Environment Chamber: Distance Test, Flow Rate Inhalation and Reproducibility Tests}

The concentration of particles at 3 sizes $(100,300$ and $600 \mathrm{~nm})$ transmitted by the head source is measured as a function of the distance, separating the two manikin heads (Figure 6(a)). It is found that the concentration of the particles remains uniform in the distance range of 20 and $50 \mathrm{~cm}$. In the controlled environment chamber, the transmission of particles released by the emitter is not influenced by the distance separating the two manikin heads. In the case of fine droplets in a poorly ventilated small space, distance alone seems to be insufficient in reducing coronavirus transmission.

The inhalation effect by the receptor head is analyzed at three different flowrates, 42.5, 60 and $85 \mathrm{~L} / \mathrm{min}$ (Figure 6(b)). For particles of approximately
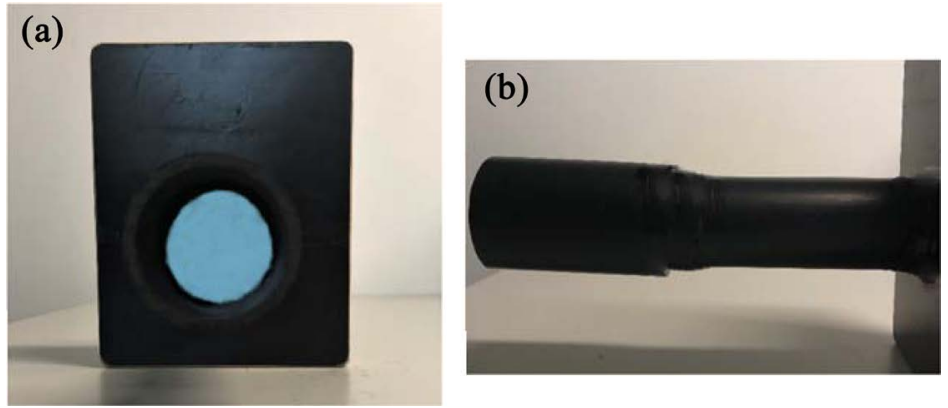

Figure 5. Material facemask holder. 


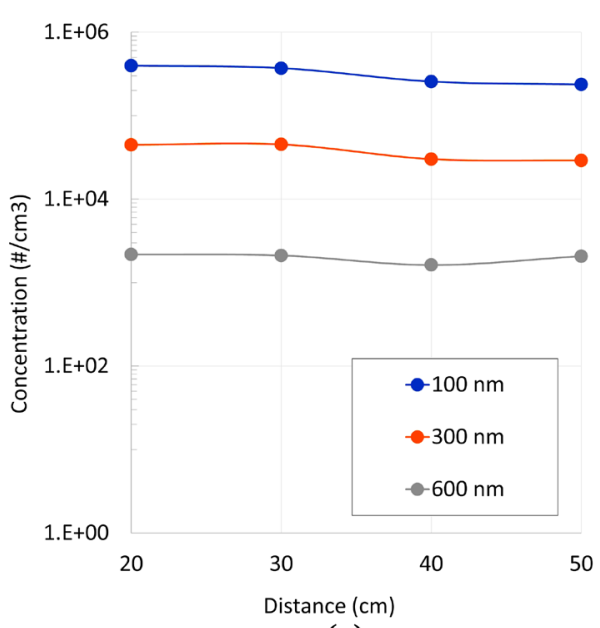

(a)

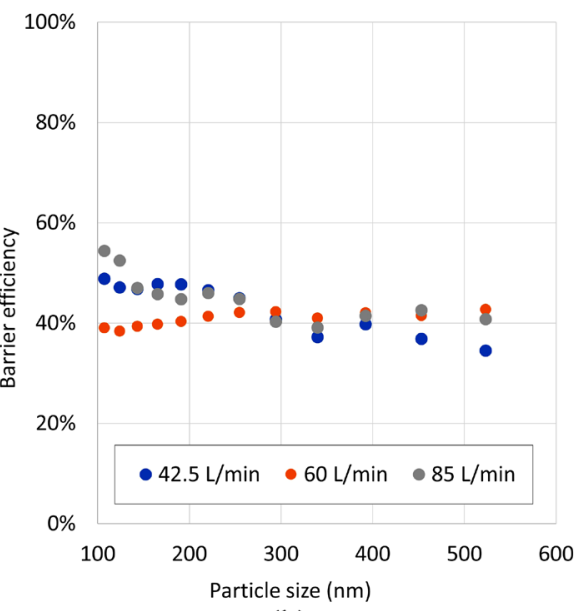

(b)

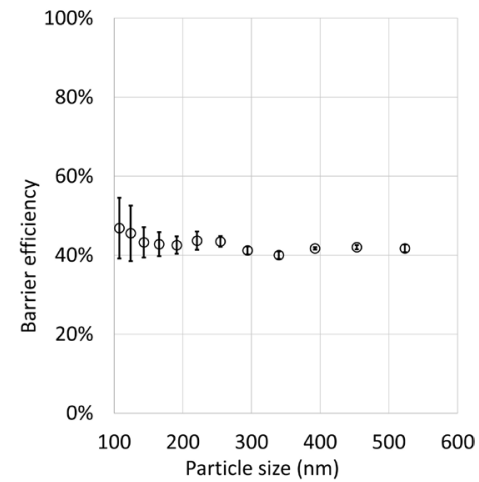

(c)

Figure 6. (a) Aerosol concentration (at $100 \mathrm{~nm}, 300 \mathrm{~nm}$ and $600 \mathrm{~nm}$ ) according to the distance between the two heads installed in the chamber; (b) Green facemask barrier efficiency in the controlled environment chamber according to the particle size measured at three different flowrates; (c) Green facemask barrier in the controlled environment chamber mean efficiency ( \pm one standard deviation) according to the particle size measured at $85 \mathrm{~L} / \mathrm{min} .(\mathrm{N}=3)$.

$100 \mathrm{~nm}$ in size, the inhalation flowrate slightly increases the barrier efficiency in the test chamber. Small aerosols (around $100 \mathrm{~nm}$ ) are carried more by the airflow. The greater the inhalation flowrate, the greater the intake of outside air into the chamber. Consequently, the dilution of the concentration of the particle transmission is great and subsequently the barrier efficiency increases. In contrast, the inhalation flowrate does not have a big influence for particle sizes greater than $200 \mathrm{~nm}$.

The data reported in this paper were the result from a single measurement. However, tests have been conducted to estimate the reproducibility for one of the facemasks. Three barrier efficiency tests were performed with the green facemask. In Figure 6(c), one can note that the standard deviation is less than 10\% of the whole curve. It can be assumed that the standard deviations measured in this situation can be applied to all configurations of facemasks presented in the "result and discussion" section. This statement is supported by the design similarities of the facemasks and by the use on the same manikin heads.

\section{Results and Discussions}

\subsection{Barrier Efficiency of the 3 Facemasks}

Figure 7 shows the barrier effectiveness of the three facemasks in the test chamber. The distance between the two heads is $20 \mathrm{~cm}$. The efficiency barrier includes both the effect of facemask materials and leakage due to facemask fitting on the emitter head.

The white facemask offers a barrier efficiency between $70 \%$ and $50 \%$.

The blue facemask offers a barrier efficiency between $80 \%$ and $50 \%$. This barrier efficiency is lower than the one associated with the white facemask. This difference could be due to the material of the facemask or to the fitting of the facemask 


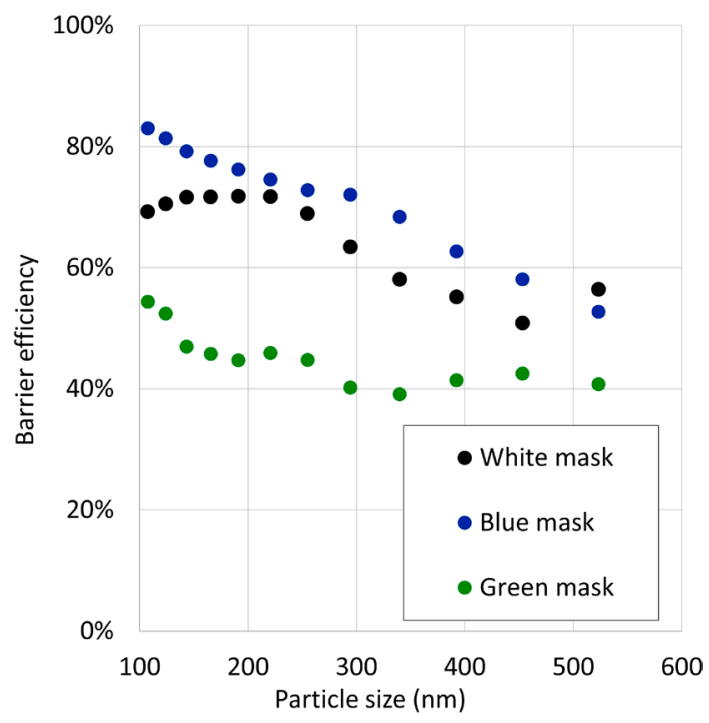

Figure 7. Barrier efficiency in the controlled environment chamber according to the particle size measured for the three facemasks measured at $85 \mathrm{~L} / \mathrm{min}$.

on the manikin head.

Finally, the green facemask offers a barrier efficiency between $60 \%$ and $40 \%$. This last result shows that the barrier efficiency in the test chamber is lower than that performed in the ventilated room (Figure 4). In the ventilated room, particles not blocked by the green facemask disperse in all directions in the chamber and are diluted by the ventilation airflow. Transmission is then less important in the ventilated, open-environment room than in the confined environment without ventilation.

In Figure 7, one can observe that the green facemask offers less barrier efficiency than the blue and the white ones. However, the difference between the results obtained by the three material facemasks is not significant.

\subsection{Leakage Related to Fitting of the 3 Facemasks}

To study the intrinsic impact of facemask materials on barrier efficiency, the facemasks are sealed on the facemask wearer to eliminate leaks due to fitting. The results obtained are then compared to those without sealing (Figure 8).

The materials of the white and blue facemasks effectively block the particles emitted by the source. The barrier efficiency is in the order of 100\% (Figure 8(a) and Figure $8(\mathrm{~b})$ ). This efficiency quickly drops when the facemasks are not sealed on the head wearer. Barrier efficiency is therefore influenced by leaks due to fitting and not by the material of the white and blue facemasks.

The same result is observed for the green material of the textile facemask for particles larger than $500 \mathrm{~nm}$ (Figure 8(c)). The effect of leakage decreases significantly for particle sizes below $500 \mathrm{~nm}$. However, the barrier efficiency of the sealed green facemask is greater than that the unsealed one. It is greater than $70 \%$ for particles of size less than $500 \mathrm{~nm}$ and it reaches a barrier efficiency of $90 \%$ for particles of size greater than $500 \mathrm{~nm}$. 
The barrier efficiency appears to decrease when the particle size increases from $100 \mathrm{~nm}$ to $500 \mathrm{~nm}$, and this trend is more pronounced for commercial facemasks than for homemade facemasks.

In conclusion, the barrier efficiency is more affected by fitting than the material of the facemask. These results show that the principle effect on the performance barrier of facemasks are breathability comfort (material pressure drop) and a design that provides the best fit for minimizing leakage. The impact of the filtration efficiency of the material is not a major factor for facemask qualifications.

\subsection{Leakage Related to Holes in the 3 Facemasks}

Figure 9 presents the barrier efficiency according to the particle size measured using the material facemask holder (Figure 5) for the three different materials.

Figure 9(a) shows the effect of the holes on the efficiency barrier of the white facemask material. Without holes, the material performs very well, and its

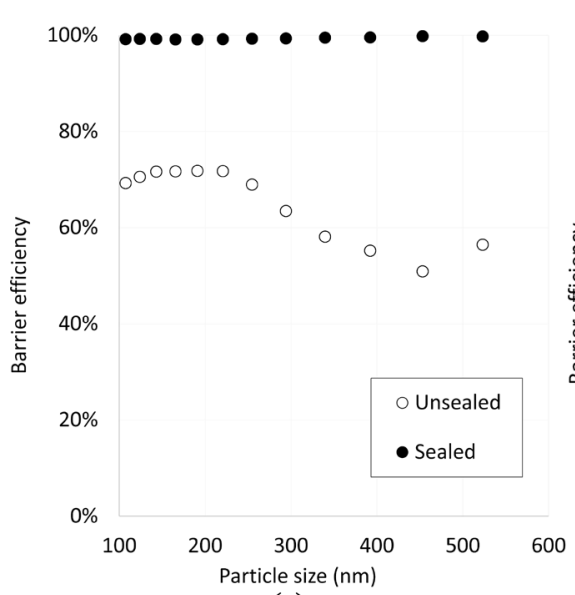

(a)

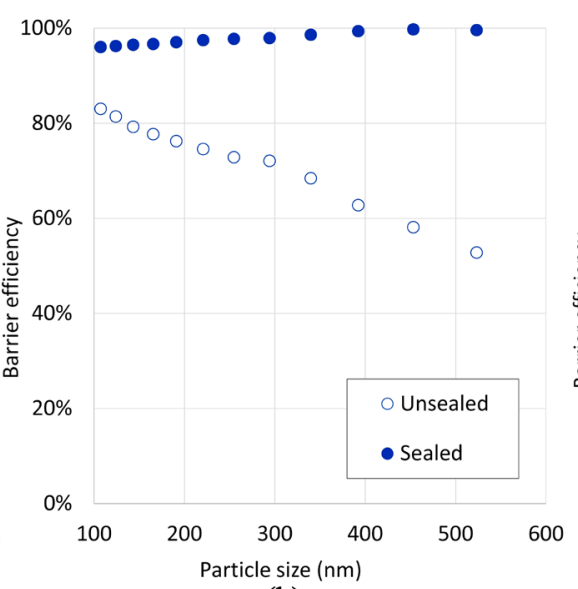

(b)

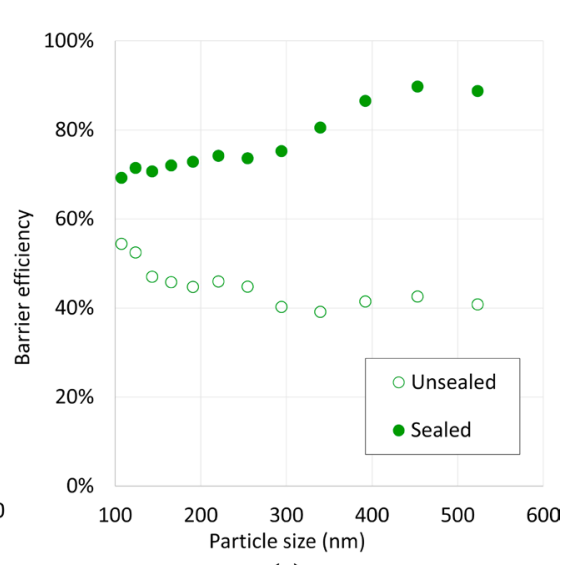

(c)

Figure 8. Barrier efficiency of sealed and unsealed facemasks in the controlled environment chamber according to the particle diameter: (a) for the white facemask, (b) for the blue facemask and (c) for the green facemask, measured at $85 \mathrm{~L} / \mathrm{min}$.

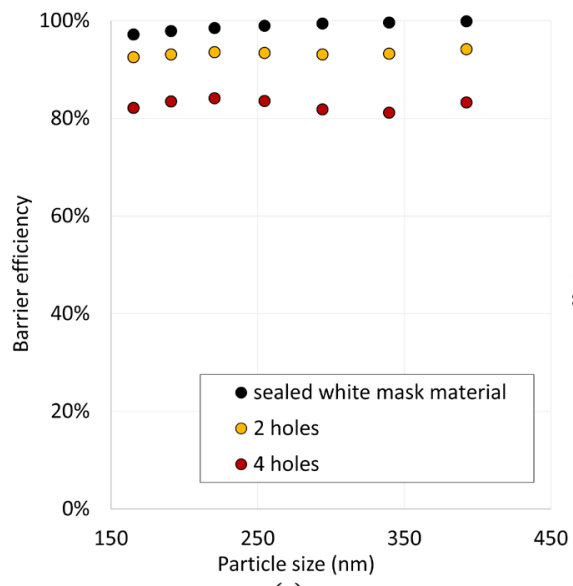

(a)

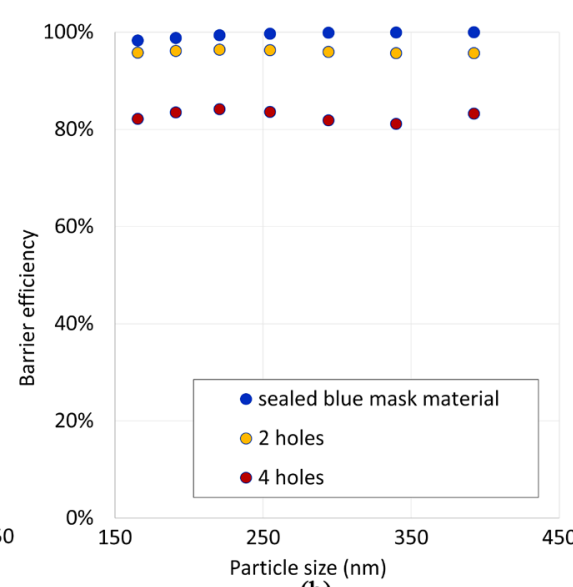

(b)

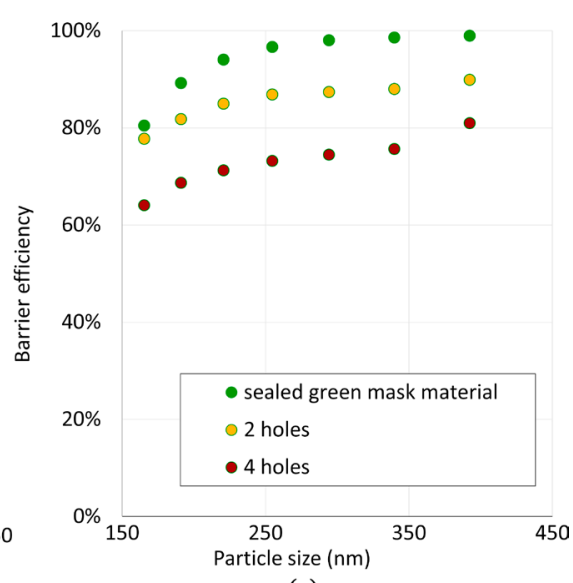

(c)

Figure 9. Barrier efficiency of the intact material facemasks and with holes according to particle size: (a) for the white facemask material, (b) for the blue facemask material and (c) for the green facemask material, measured at $85 \mathrm{~L} / \mathrm{min}$. 
barrier efficiency is near $100 \%$. With the presence of holes this efficiency continues to decrease as more holes are drilled. Figure 9(b) shows the effect of the holes on the efficiency barrier of the blue facemask material. Similar observations of the white facemask material were observed. Figure 9(c) shows the effect of the holes on the efficiency barrier of the green facemask of woven cotton material. The barrier efficiency increases with the size of the particles projected to reach a maximum plateau for particle sizes greater than $300 \mathrm{~nm}$. The textile material offers a barrier efficiency greater than $80 \%$ and reaches the value of $100 \%$ without holes. These results agree with the studies of Chen and Willeke [21], Liu et al. [32] on FFRs and Mouret et al. [33] on filter media. These authors showed that above a specific hole size, the protection factor decreases very rapidly with increasing leakage size. In addition, Rengasamy and Eimer published two studies [34] [35] presenting the same conclusions for N95 FFRs. They also observed that FFRs with lower efficiency when sealed have higher protection factor that the other FFRs. Then the barrier efficiency is reduced in the presence of the holes or valves. These results confirm those obtained in the previous section that leaks have a major effect on the barrier effectiveness of the facemask. These results suggest that the facemask should be manufactured in such a way that leaks are minimized by emphasizing fit in the mask design and avoiding holes in its manufacture.

\section{Conclusions}

Since the transmission of SARS-CoV-2 by the aerosol route has been established and acknowledged by the WHO and many governments, the scientific community must work on the evaluation and qualification of facemasks. Unlike the masks conventionally used as respiratory protective devices and surgical masks, facemasks in use by the general population are still little studied, and do not currently have a standard stating their qualifications. Although it is possible to learn from the established standards for conventional masks, it is necessary to develop a new expertise and a methodology specific to these facemasks. This paper demonstrates the need for a different methodology for this type of facemask due to their specific use and design.

The results presented above have shown that a facemask, although less effective than an FFR or a surgical mask, can reduce the aerosol concentration downstream of the mask. The results also demonstrate that the effect of leaks on the face/mask interface drastically reduces the barrier efficiency of the facemasks studied here. In addition to the filtration efficiency and pressure drop, facial leakage plays a prominent role in the barrier performance of these masks in their ability to stop viral spread. The fit of the mask to the head must be optimal, and the design must limit the presence of side leakage, holes or valves. Each type of facemask has its own advantages, e.g. commercial facemasks are easier to wear for a long time because of breathability, but fabric facemasks have the advantage of being more environmentally friendly. Performance tests for facemask need to 
be deeply studied, defined more globally and classified according to their use

(e.g. at work or for shopping).

\section{Acknowledgements}

The authors would like to acknowledge the IRSST (Institut de recherché Robert Sauvé en Santé et en sécurité du Travail) for the financial support of this work.

\section{Conflicts of Interest}

The authors declare no conflicts of interest regarding the publication of this paper.

\section{References}

[1] National Academies of Sciences, Engineering, and Medicine (2020) Rapid Expert Consultation on the Possibility of Bioaerosol Spread of SARS-CoV-2 for the COVID-19 Pandemic (April 1, 2020). The National Academies Press, Washington DC.

[2] Morawska, L. and Milton, D.K. (2020) Open Letter to World Health Organization. Clinical Infectious Diseases Journal, July 8, 2020.

[3] World Health Organization (WHO) (2020) Transmission of SARS-CoV-2-Implications for Infection Prevention Precaution.

https://www.who.int/news-room/commentaries/detail/transmission-of-sars-cov-2-i mplications-for-infection-prevention-precautions

[4] Health Canada (2020) Non-Medical Masks and Face Coverings: About. https://www.canada.ca/en/public-health/services/diseases/2019-novel-coronavirus-i nfection/prevention-risks/about-non-medical-masks-face-coverings.html

[5] Government of Quebec (2020) Wearing a Mask or a Face Covering in Public Settings in the Context of the COVID-19 Pandemic.

https://www.quebec.ca/en/health/health-issues/a-z/2019-coronavirus/wearing-a-fac e-covering-in-public-settings-in-the-context-of-the-covid-19-pandemic

[6] UK Government (2020) Guidance-Face Coverings: When to Wear One and How to Make Your Own.

https://www.gov.uk/government/publications/face-coverings-when-to-wear-one-an d-how-to-make-your-own/face-coverings-when-to-wear-one-and-how-to-make-yo ur-own

[7] Lyu, W. and Wehby, G.L. (2020) Community Use of Face Masks and COVID-19: Evidence from a Natural Experiment of State Mandates in the U.S. Health Affairs Journal, 39, 1419-1425. https://doi.org/10.1377/hlthaff.2020.00818

[8] Health Canada (2020) COVID-19 Medical Masks and N95 Respirators-Overview. https://www.canada.ca/en/health-canada/services/drugs-health-products/medical-d evices/masks-respirators-covid19.html

[9] Code of Federal Regulations (CFR) (1995) 42 CFR, Part 84 Respiratory Protection Devices. US Government Printing Office, Washington DC.

[10] Rengasamy, S., Eimer, B. and Shaffer, R.E. (2010) Simple Respiratory Protection-Evaluation of the Filtration Performance of Cloth Masks and Common Fabric Materials against 20-1000 nm Size Particles. Annals of Occupational Hygiene, 7, 789.

[11] Davies, A., Thompson, K.-A., Giri, K., Kafatos, G., Walker, J. and Bennett, A. (2013) 
Testing the Efficacy of Homemade Masks: Would They Protect in an Influenza Pandemic? Disaster Medicine and Public Health Preparedness, 7, 413. https://doi.org/10.1017/dmp.2013.43

[12] Konda, A., Prakash, A., Moss, G.A., Schmoldt, M., Grant, G.D. and Guha, S. (2020) Aerosol Filtration Efficiency of Common Fabrics Used in Respiratory Cloth Masks. ACS Nano, 14, 6339. https://doi.org/10.1021/acsnano.0c03252

[13] National Academies of Sciences, Engineering, and Medicine (2020) Rapid Expert Consultation on the Effectiveness of Fabric Masks for the COVID-19 Pandemic (April 8, 2020). The National Academies Press, Washington DC. https://doi.org/10.17226/25776

[14] Neupane, B.B., Mainali, S., Sharma, A. and Giri, B. (2019) Optical Microscopic Study of Surface Morphology and Filtering Efficiency of Face Masks. Peer), 7, e7142. https://doi.org/10.7717/peerj.7142

[15] Fisher, E.P., Fischer, M.C., Grass, D., Henrion, I., Warren, W.S. and Westman, E. (2020) Low-Cost Measurement of Facemask Efficacy for Filtering Expelled Droplets during Speech. Science Advances, 6, eabd3083. https://doi.org/10.1101/2020.06.19.20132969

[16] Aydin, O., Emon, B., Cheng, S., Hong, L., Chamorro, L.P. and Saif, M.T.A. (2020) Performance of Fabrics for Home-Made Masks against the Spread of COVID-19 through Droplets: A Quantitative Mechanistic Study. Extreme Mechanics Letters, 40, Article ID: 100924. https://doi.org/10.1016/j.eml.2020.100924

[17] Centers for Disease Control and Prevention (CDC) (2020) How to Make Cloth Face Covering.

https://www.cdc.gov/coronavirus/2019-ncov/prevent-getting-sick/how-to-make-clo th-face-covering.html

[18] Association Française de Normalisation (AFNOR) (2020) AFNOR SPEC S76-001Masques barrières-Guide d'exigences minimales, de méthodes d'essais, de confection et d'usage.

[19] ASTM International (2021) F3502-21 Standard Specification for Barrier Face Coverings. ASTM International, West Conshohocken.

[20] Hinds, W.C. and Kraske, G. (1987) Performance of Dust Respirators with Facial Seal Leaks I. Experimental. American Industrial Hygiene Association Journal, 48, 836. https://doi.org/10.1080/15298668791385679

[21] Chen, C.C. and Willeke, K. (1992) Characteristics of Face Seal Leakage in Filtering Facepieces. American Industrial Hygiene Association Journal, 53, 533. https://doi.org/10.1080/15298669291360120

[22] Brochot, C., Michielsen, N., Chazelet, S. and Thomas, D. (2012) Measurement of Protection Factor for Respiratory Protective Devices toward Nanoparticles. The Annals of Occupational Hygiene, 5, 595.

[23] Van Der Sande, M., Teunis, P. and Sabel, R. (2008) Professional and Home-Made Face Masks Reduce Exposure to Respiratory Infections among the General Population. PLoS ONE, 3, e2618. https://doi.org/10.1371/journal.pone.0002618

[24] Bahloul, A., Mahdavi, A., Haghighat, F. and Ostiguy, C. (2014) Evaluation of N95 Filtering Facepiece Respirator Efficiency with Cyclic and Constant Flows. Journal of Occupational and Environmental Hygiene, 11, 499. https://doi.org/10.1080/15459624.2013.877590

[25] Mahdavi, A., Bahloul, A., Haghighat, F. and Ostiguy, C. (2014) Contribution of Breathing Frequency and Inhalation Flow Rate on Performance of N95 Filtering Facepiece Respirators. The Annals of Occupational Hygiene, 58, 195. 
[26] Mahdavi, A., Haghighat, F., Bahloul, A., Brochot, C. and Ostiguy, C. (2015) Particle Loading Time and Humidity Effects on the Efficiency of an N95 Filtering Facepiece Respirator Model under Constant and Inhalation Cyclic Flows. The Annals of Occupational Hygiene, 59, 629.

[27] Brochot, C., Djebara, A., Haghighat, F. and Bahloul, A. (2015) Validation of a Laboratory Test Bench for the Efficiency of an N95 Filtering Face Piece, Using Simulated Occupational Exposure. Journal of Environmental and Analytical Toxicology, 5, 286. https://doi.org/10.4172/2161-0525.1000286

[28] Brochot, C., Haghighat, F. and Bahloul, A. (2020) Comparative Performance of the NanoScan and the Classic SMPS in Determining N95 Filtering Facepiece Efficiency against Nanoparticles. Aerosol Science and Engineering, 4, 178. https://doi.org/10.1007/s41810-020-00064-4

[29] Tang, J.W., Nicolle, A.D., Klettner, C.A., Pantelic, J., Wang, L., Suhaimi, A.S., Tan A.Y.L., Ong, G.W.X., Su, R., Sekhar, C., Cheong, D.D.W. and Tham, K.W. (2013) Airflow Dynamics of Human Jets: Sneezing and Breathing-Potential Sources of Infectious Aerosols. PLoS ONE, 8, e59970.

https://doi.org/10.1371/journal.pone.0059970

[30] Chao, C.Y.H., Wan, M.P., Morawaska, L., Johnson, G.R., Ristovski, Z.D., Hargreaves, M., Mengersen, K., Corbett, S., Li, Y., Xie, X. and Katoshevshi, D. (2009) Characterization of Expiration Air Jets and Droplet Size Distributions Immediately at the Mouth Opening. Aerosol Science, 40, 122. https://doi.org/10.1016/j.jaerosci.2008.10.003

[31] Bourouiba, L., Dehandschoewercker, E. and Bush, J.W.M. (2014) Violent Expiratory Events: On Coughing and Sneezing. Journal of Fluid Mechanics, 745, 537. https://doi.org/10.1017/jfm.2014.88

[32] Liu, B.Y.H., Lee, J.K., Mullins, H. and Danish, S.G. (1993) Respirator Leak Detection by Ultrafine Aerosols: A Predictive Model and Experimental Study. Aerosol Science and Technology, 19, 15. https://doi.org/10.1080/02786829308959617

[33] Mouret, G., Thomas, D., Chazelet, S., Appert-Collin, J.C. and Bemer, D. (2009) Penetration of Nanoparticles through Fibrous Filters Perforated with Defined Pinholes. Journal of Aerosol Science, 40, 762. https://doi.org/10.1016/j.jaerosci.2009.04.010

[34] Rengasamy, S. and Eimer, B.C. (2011) Total Inward Leakage of Nanoparticles through Filtering Facepiece Respirators. The Annals of Occupational Hygiene, 55, 253.

[35] Rengasamy, S. and Eimer, B.C. (2012) Nanoparticle Penetration through Filter Media and Leakage through Face Seal Interface of N95 Filtering Facepiece Respirators. The Annals of Occupational Hygiene, 56, 568. 